\title{
Rupture of an Infrarenal Aortic Aneurysm Due to a Secondary Type 1a Endoleak Following Endovascular Aneurysm Sealing with the Nellix ${ }^{\circledR}$ Device
}

\author{
Kudla Dorothea1, Uhl Christian1, Zorger Niels², Steinbauer Markus', Topel Ingolf ${ }^{*}$ \\ ${ }^{1}$ Department of Vascular Surgery, Krankenhaus Barmherzige Brüder Regensburg, Regensburg, Germany \\ ${ }^{2}$ Department of Diagnostic and Interventional Radiology, Neuroradiology and Nuclear Medicine, Krankenhaus Barmherzige \\ Brüder Regensburg, Regensburg, Germany \\ Email: dorothea.kudla@barmherzige-regensburg.de, christian.uhl@barmherzige-regensburg.de, \\ niels.zorger@barmherzige-regensburg.de, markus.steinbauer@barmherzige-regensburg.de, \\ *ingolf.toepel@barmherzige-regensburg.de
}

How to cite this paper: Dorothea, K., Christian, U., Niels, Z., Markus, S. and Ingolf, T. (2019) Rupture of an Infrarenal Aortic Aneurysm Due to a Secondary Type 1a Endoleak Following Endovascular Aneurysm Sealing with the Nellix Device. Surgical Science, 10, 398-404.

https://doi.org/10.4236/ss.2019.1011044

Received: March 3, 2017

Accepted: November 4, 2019

Published: November 7, 2019

Copyright $\odot 2019$ by author(s) and Scientific Research Publishing Inc. This work is licensed under the Creative Commons Attribution International License (CC BY 4.0).

http://creativecommons.org/licenses/by/4.0/

(c) (i) Open Access

\begin{abstract}
Purpose: The Nellix endovascular aneurysm sealing (EVAS) system was introduced in 2010 for infrarenal abdominal aortic aneurysm (AAA) repair. So far one secondary type 1a endoleak resulting in rupture of the aneurysm has been reported. Case report: We present a case of a rupture of an infrarenal aortic aneurysm due to a secondary type 1a endoleak caused by stent migration 14 months after endovascular aneurysm sealing with a Nellix system. Conclusion: Strict adherence to anatomical requirements given by the instructions for use during patient selection and thorough post interventional surveillance may reduce the probability of secondary type 1a endoleaks. Furthermore, device design changes, especially concerning the configuration of the proximal part of the stent graft, have to be considered.
\end{abstract}

\section{Keywords}

Endovascular Aneurysm Sealing, Secondary Type 1a Endoleak, Rupture, Abdominal Aortic Aneurysm

\section{Introduction}

Nellix (Endologix, Irvine, CA, USA) is an endovascular aneurysm sealing (EVAS) system introduced in 2010 to overcome certain difficulties associated with endovascular aneurysm repair (EVAR) of abdominal aortic aneurysms 
(AAA) [1]. The Nellix system consists of two balloon expandable stents providing the blood flow lumen through the aneurysm to the iliac arteries, each surrounded by a polyurethane endobag which is inflated and filled with a polymer sealing the aneurysmal sac to prevent retrograde blood flow and to stabilize the stents to avoid stent migration [2]. So far only a few postoperative complications leading to conversion to surgery or rupture have been reported [3] [4] [5] [6].

In 2016 we observed for the first time a rupture of an infrarenal aortic aneurysm due to a secondary type 1a endoleak in a patient who had undergone EVAS using a Nellix system. This paper is to share the case and to discuss the possible causes and consequences.

\section{Case Report}

In November 2014, an 80-year-old male patient was admitted to our institution with acute epigastric pain. Ultrasound and CT Angiography revealed a progression of his known infrarenal aortic aneurysm (diameter increase of $3 \mathrm{~cm}$ in 3 years, maximal diameter $56 \mathrm{~mm}$ ). The patient had a history of arterial hypertension, coronary artery disease and chronic renal failure. A few years ago a right hemicolectomy had been done due to colon cancer.

Ultrasound and computed tomography angiography (CTA) showed an infrarenal aortic aneurysm with a maximal diameter of $56 \mathrm{~mm}$ and an infrarenal neck length of $8 \mathrm{~mm}$ (Figure 1). Due to aneurysm morphology and history of open abdominal surgery we decided to implant a Nellix EVAS device, which was inserted under general anesthesia without complication at the same day. At the end of the procedure digital subtraction angiography proved a correct position of the grafts and no evidence of leak, rupture or dissection (Figure 2).

There were no further postoperative complications and the patient was discharged 10 days later.

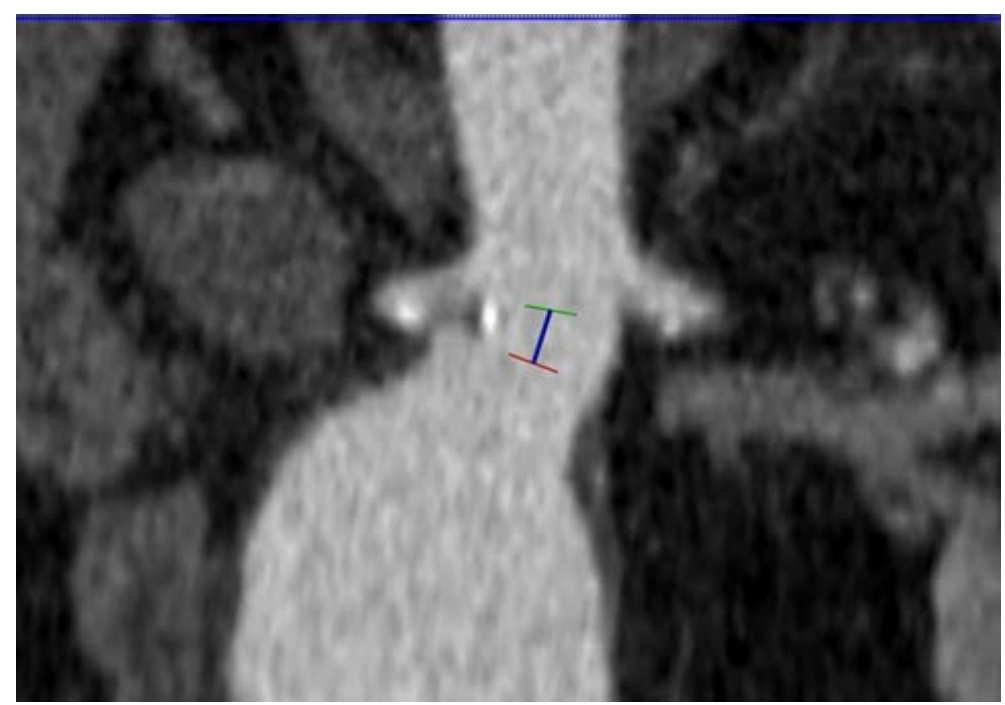

Figure 1. Preoperative CT-angiography showing the aneurysm neck in a centerline reconstruction with a length of $8.2 \mathrm{~mm}$. 


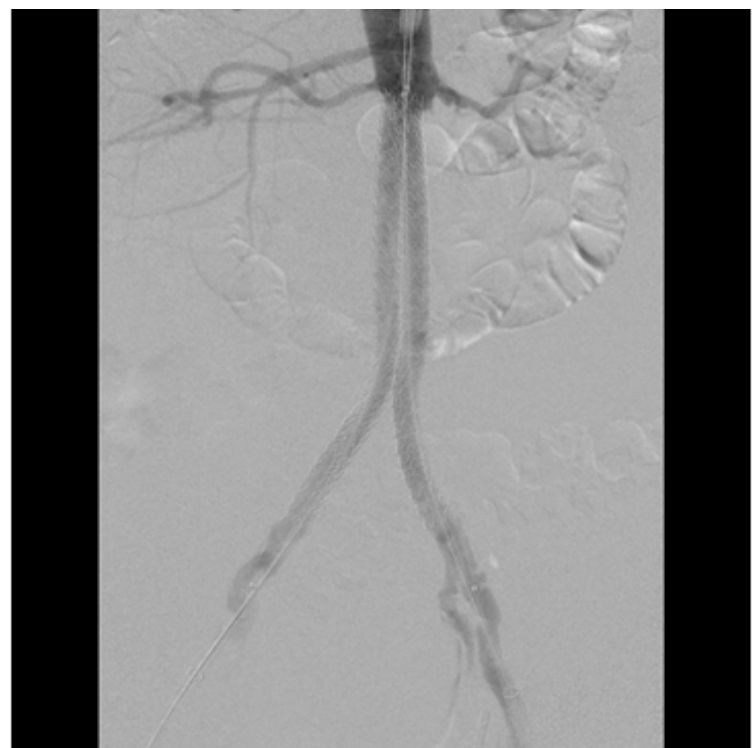

Figure 2. Intraoperative completion angiogram with the Nellix device placed correctly. No Endoleak.

At 2 months follow-up the asymptomatic patient's CT scan showed precise positioning of the endograft without endoleak or increasing size of the AAA (Figure 3). Sagittal planes already showed a discrete ventral migration of the proximal part of the stentgraft. Unfortunately, the patient did not show up for scheduled controls at 6 and 12 months.

One year later the patient was re-admitted to our institution with progressive abdominal and back pain. His blood pressure was 160/80 $\mathrm{mmHg}$, pulse $62 \mathrm{bpm}$, Hemoglobin $13.4 \mathrm{~g} / \mathrm{dl}$. On clinical examination a pulsatile abdominal mass was palpable. Sonography showed progression of the aneurysm size to a maximal diameter of $65 \mathrm{~mm}$. This was confirmed by the immediately following CTA, which also revealed a rupture of the AAA with a large retroperitoneal hematoma. Rupture was caused by a proximal type 1 endoleak due to caudal and ventral dislocation of the proximal stent grafts (Figure 4).

After laparotomy extensive adhesions from the previous operation made it difficult to enter the retroperitoneal space. After suprarenal clamping and dissection of the aneurysm sac it became obvious that the Nellix stents had torn open the anterior aneurysm wall. We opened the aneurysm sac and removed the Nellix device. Due to limitation of the distal preparation of the aneurysm caused by pronounced cicatrization following preceding surgical procedures we implanted a tube graft (Maquet Synergy $20 \mathrm{~mm}$ ). Owing to a residual dissection of the left common iliac artery seen in the intraoperative digital subtraction angiography a covered stent (BARD Fluency $10 \times 60 \mathrm{~mm}$ ) was inserted. Blood loss was $1800 \mathrm{ml}$, transfusion of 3 packed red blood cells, 6 fresh frozen plasma and $350 \mathrm{ml}$ cell saver salvaged blood was required.

After initially stable vital signs after surgery the patient became hemodynamically instable again and blood loss through the drain some hours postoperatively 


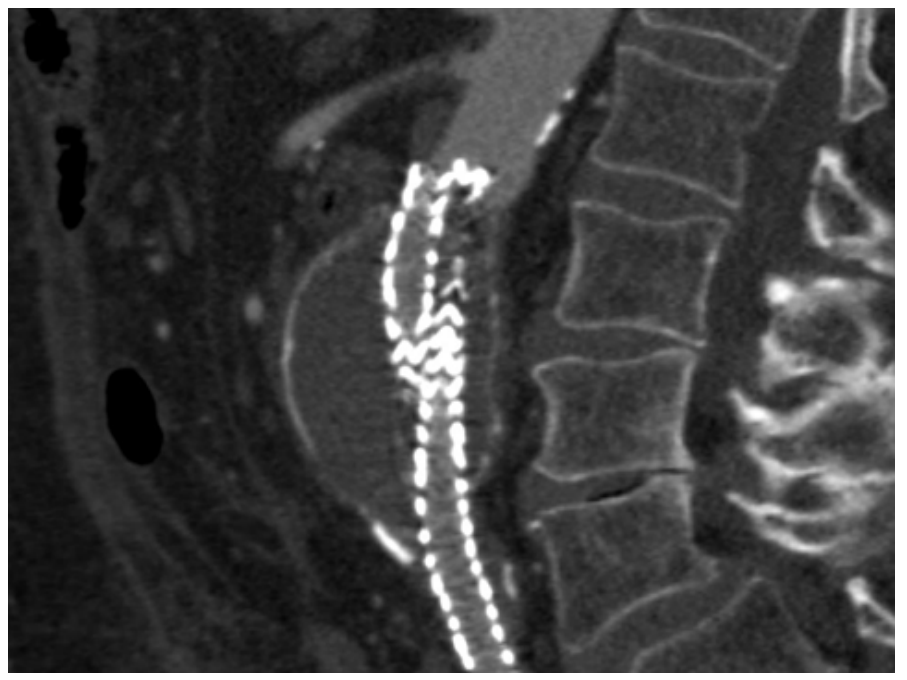

Figure 3. First postoperative CT-angiography at 2 months. Reconstructions already show a discrete ventral migration of the proximal part of the stents but yet no endoleak.

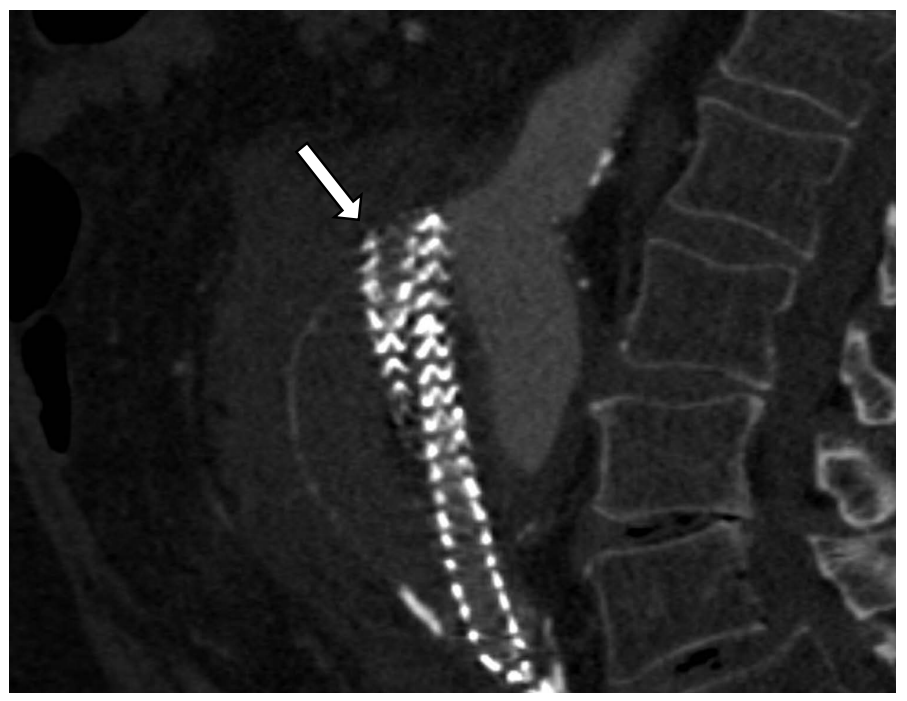

Figure 4. CT-angiography 14 months after implantation. Loss of proximal fixation led to secondary type 1a endoleak. Proximal ends of the stents perforated the aneurysm wall causing rupture (arrow).

occurred. In the following abdominal revision surgery there was diffuse bleeding in the entire abdomen. We aimed for a temporary abdominal closure with abdominal packing. But despite massive transfusion of erythrocytes, fresh frozen plasma and thrombocytes the patient became increasingly hemodynamically unstable and was requiring high doses of catecholamines during the procedure. Disseminated intravascular coagulation (DIC) caused progressive diffuse bleeding and intravasal thrombosis resulting in occlusion of the tube and the iliac, femoral and popliteal arteries with critical ischemia of the lower body. As it was not possible to permanently recanalize the tube or the iliac, femoral or popliteal arteries by thrombectomy we did not extend therapy at this point in interdiscip- 
linary consensus. The patient died some hours later at the intensive care unit.

\section{Discussion}

EVAR has become a mainstay of AAA treatment [7]. EVAS technology was introduced to overcome certain problems with conventional endografts such as device migration and secondary sac progression caused by type 1 and 2 endoleaks. The importance of neck quality to the outcome of endovascular therapy of AAA has been extensively discussed [8]. Although the instructions for use of the Nellix device recommend a minimal neck length of $10 \mathrm{~mm}$ successful experiences with shorter necks were reported [1] [9].

Our case demonstrates that secondary device migration might occur especially in patients with aortic necks shorter than $10 \mathrm{~mm}$. Furthermore, the rigidity of the balloon-expandable stents led to ventral dislocation and sac perforation after loss of the proximal landing zone.

During a $15+/-6$ months follow-up of an initial clinical trial experience involving 34 patients no changes in sideways or longitudinal position of the endograft within the aneurysm sac were seen. Furthermore, no secondary endoleaks, no conversion to surgery and no aneurysm rupture were reported [10]. Two limited-space type 1 endoleaks occurred: A postprocedural type 1a endoleak (seen on the 1-month CT scan) resolved spontaneously after two months (and remained sealed in CT scan after 6 and 12 months). A type $1 \mathrm{~b}$ endoleak occurring at the time of intervention (due to a long aneurysm sac and non-availability of Nellix iliac extenders in the early clinical experience) was stable in size (in CT scan after 1, 6 and 12 months) and was resoluted in an elective secondary endovascular procedure at 15 months [9] [10]. In a single center study including 50 EVAS procedures and evaluating amongst others device related complications one patient suffered from a secondary type 2 endoleak which did not demand a secondary intervention [6].

Ameli-Renani and co-workers reported a type 1a endoleak 4 days after Nellix EVAS which was successfully eliminated by endovascular embolization [11]. A case of delayed recurrence of type 1a endoleak resulting in rupture of the aorta and retroperitoneal hemorrhage six months after EVAS was published in 2016 [5]. Another patient showing separation of the endobags and mild enlargement of the aneurysmal sac in the two-month post-EVAS CT scan without detectable endoleaks developed a type 1a endoleak complicated by rupture of the AAA four months after Nellix implantation [4].

It is known that primary type 1a endoleaks can be caused by incorrect low deployment of the Nellix devices or inadequate filling of the endobags and become larger with time when untreated [12].

In our case the secondary type la endoleak seems to be provoked by stent migration and dislocation of the proximal stent out of the neck. The rigidity of the stent and the angulation lead to an enhanced pressure to the anterior aortic wall and rupture. This leads to the assumption that filling the aneurysm sac does not 
provide sufficient long-term positional stability to the endograft to prevent migration. This counts against the extension of the time interval for follow-up as proposed in some publications [13] and requires improvement of the configuration of the proximal part of the stent graft to prevent a perforation of the aortic wall.

\section{Conclusions}

This case shows that using the EVAS technology does not exclude the secondary occurrence of proximal type 1a endoleaks. Shortness and angulation of proximal neck might promote device dislocation. Due to the rigidity of the stents perforation of the ventral aneurysm wall can occur.

Accurate patient election and close postoperative surveillance of patients undergoing Nellix EVAS are mandatory.

\section{Conflicts of Interest}

The authors declare that they have no competing interests.

\section{References}

[1] Böckler, D., Reijnen, M.M., Krievins, D., et al. (2014) Use of the Nellix EVAS System to Treat Post-EVAR Complications and to Treat Challenging Infrarenal Necks. The Journal of Cardiovascular Surgery, 55, 601-612.

[2] Donayre, C.E., Zarins, C.K., Krievins, D.K., et al. (2011) Initial Clinical Experience with a Sac-Anchoring Endoprosthesis for Aortic Aneurysm Repair. Journal of Vas cular Surgery, 53, 574-582. https://doi.org/10.1016/j.jvs.2010.09.009

[3] Carpenter, J.P., Cuff, R., Buckley, C., et al. (2016) Results of the Nellix System Investigational Device Exemption Pivotal Trial for Endovascular Aneurysm Sealing. Journal of Vascular Surgery, 63, 23-31. https://doi.org/10.1016/j.jvs.2015.07.096

[4] Cheng, L.F., Cheung, K.F., Chan, K.M., et al. (2016) Early Enlargement of Aneurysmal Sac and Separation of EndoBags of Nellix Endovascular Aneurysm Sealing System as Signs of Increased Risk of Later Aneurysm Rupture. CardioVascular and Interventional Radiology, 39, 1654-1657.

https://doi.org/10.1007/s00270-016-1416-0

[5] Pua, U., Quek, L.H. and Tan, G.W. (2016) Delayed Recurrence of Type 1A Endoleak with Aortic Rupture and Hemorrhage after Endovascular Aneurysm Sealing (EVAS). Cardio Vascular and Interventional Radiology, 39, 1061-1065. https://doi.org/10.1007/s00270-015-1291-0

[6] Zerwes, S., Nurzai, Z., Leissner, G., et al. (2016) Early Experience with the New Endovascular Aneurysm Sealing System Nellix: First Clinical Results after 50 Implantations. Vascular, 24, 339-347. https://doi.org/10.1177/1708538115605430

[7] Schmitz-Rixen, T., Torsello, G., Steinbauer, M., et al. (2016) The Endovascular Performance Spectrum of Vascular Surgery Departments in Germany. Gefäßchirurgie, 21, 570-663. https://doi.org/10.1007/s00772-016-0157-3

[8] Oikonomou, K., Katsargyris, A. and Verhoeven, E. (2014) Indikationen zur primären oder sekundären Implantation von fenestrierten Stentprothesen beim hostile AAA neck. Gefäßchirurgie, 19, 220-225.

https://doi.org/10.1007/s00772-013-1276-8 
[9] Navarro, T.P., de Castro Bernardes, R., Procopio, R.J., et al. (2014) Treatment of Hostile Proximal Necks during Endovascular Aneurysm Repair. AORTA, 2, 28. https://doi.org/10.12945/j.aorta.2014.13-030

[10] Krievins, D.K., Holden, A., Savlovskis, J., et al. (2011) EVAR Using the Nellix Sac-Anchoring Endoprosthesis: Treatment of Favourable and Adverse Anatomy. European Journal of Vascular and Endovascular Surgery, 42, 38-46. https://doi.org/10.1016/j.ejvs.2011.03.007

[11] Ameli-Renani, S., Das, R., Weller, A., et al. (2015) Embolisation of a Proximal Type I Endoleak Post-Nellix Aortic Aneurysm Repair Complicated by Reflux of Onyx into the Nellixendograft Limb. CardioVascular and Interventional Radiology, 38, 747-751. https://doi.org/10.1007/s00270-014-1044-5

[12] Holden, A., Savlovskis, J., Winterbottom, A., et al. (2016) Imaging after Nellix Endovascular Aneurysm Sealing: A Consensus Document. Journal of Endovascular Therapy, 23, 7-20. https://doi.org/10.1177/1526602815616251

[13] Krievins, D., Savlovskis, J., Kisis, K., et al. (2013) Aortic Aneurysm Repair Using an Endoprosthesis Designed to Eliminate Endoleaks: Three Year Follow-Up. American Journal of Cardiology, 111, 7. https://doi.org/10.1016/j.amjcard.2013.01.323 